\section{$\underset{\substack{\text { hommes } \\ \text { \& migrations }}}{ }$}

\section{Hommes \& migrations}

Revue française de référence sur les dynamiques

migratoires

$1280 \mid 2009$

Les Turcs en France : quels ancrages?

\title{
Permanences et mutations de l'immigration turque en France
}

\section{Stéphane de Tapia}

\section{(2) OpenEdition Journals}

\section{Édition électronique}

URL : http://journals.openedition.org/hommesmigrations/286

DOI : 10.4000/hommesmigrations.286

ISSN : 2262-3353

\section{Éditeur}

Musée national de l'histoire de l'immigration

\section{Édition imprimée}

Date de publication : 1 juillet 2009

Pagination : 8-20

ISSN : 1142-852X

Référence électronique

Stéphane de Tapia, «Permanences et mutations de l'immigration turque en France », Hommes \& migrations [En ligne], 1280 | 2009, mis en ligne le 29 mai 2013, consulté le 30 avril 2019. URL : http:// journals.openedition.org/hommesmigrations/286 ; DOI : 10.4000/hommesmigrations.286 


\section{Permanences et mutations de l'immigration turque en France}

Par Stéphane de Tapia, géographe, directeur de recherche au CNRS, chargé de cours au Département d'Études turques de l'Université de Strasbourg, chercheur associé à MIGRINTER (Poitiers), chargé de mission auprès de l'Inspection générale de l'Éducation nationale pour le turc

En France, les immigrés originaires de Turquie et leurs descendants font le double pari de l'insertion économique et du maintien d'une forte cohésion communautaire. Française par le sol, cette population veut rester turque par le cœur. Cet entre-deux est-il viable ? Pour répondre, il faut replonger dans les racines de l'identité turque. Se souvenir que la République d'Atatürk a conservé de l'Empire ottoman sa diversité ethnique, confessionnelle,

linguistique et culturelle. Si dans l'Hexagone la transmission de la langue turque reste l'élément le plus évident de l'identité collective, il n'en demeure pas moins le plus fragile. De quoi démasquer bon nombre d'idées reçues. 
Il est souvent affirmé, y compris dans des appels d'offre émis par des instances publiques finançant la recherche, que l'on sait peu de choses sur l'immigration turque en France, "dernière arrivée", "encore mal connue", et de reprendre l'expression, plutôt malheureuse avec le recul, d'"exception turque", qui serait difficile, sinon impossible à assimiler, pardon (vrai-faux lapsus) à intégrer. C'est, d'une part, faire bien peu de cas des recherches effectuées en France sur ce sujet depuis le début des années quatre-vingt (les tout premiers textes datant des années soixante), d'autre part, faire l'impasse sur une présence turque ayant aujourd'hui plus de quatre décennies de développement. Mais de qui parle-t-on?

Les Turcs dont il est ici question sont en réalité bien souvent des Français par naturalisation, naissance ou autre mode d'acquisition de nationalité. Beaucoup préfèrent donc de ce fait parler non des "Turcs" mais des "originaires de Turquie" en France. Cette expression bien française reprend en la traduisant une idée turque consistant à opposer dans le discours, d'une part, Türk - ethnonyme apparu dans les montagnes aujourd'hui mongoles des Khenteï et transcrit dans les annales chinoises du Ve siècle par T'ou-Kiue (Tujue, en transcription pinyin) et, de ce fait, mal approprié lorsque l'on met en avant la diversité ethnique (kurde, arabe, arménienne, caucasienne, balkanique...) - et, d'autre part, Türkiye'li - précisément "originaire de Turquie", pour justement marquer son respect de la diversité ethnique, confessionnelle, linguistique, culturelle... qui caractérise la Turquie historique comme contemporaine. Un peu comme si l'on opposait Franc à Français...

Alors que la Turquie est candidate à l'adhésion à l'Union européenne, au-delà des polémiques sur ce dossier (que nous préférons ne pas développer ici pour ne pas devenir rapidement désagréable), il est piquant de constater dans nombre de nomenclatures officielles françaises (mais pas toutes !) que la Turquie est située dans la catégorie statistique "Autre Asie", alors que ce pays fait partie de la totalité des instances européennes, y compris les plus anecdotiques, des coupes de football et du concours Eurovision de la chanson aux élections des Miss Europe, Monde et Univers!

Les immigrés turcs et leurs descendants auraient-ils un coupable penchant à la clandestinité ? Que nenni ! Ils ont au contraire souvent milité pour la reconnaissance de leurs droits sociaux et civiques, ont à l'occasion des dernières élections municipales réussi à se placer à des postes d'élus, surtout dans le Grand Est de la France, et sont souvent désignés pour leur visibilité (création d'entreprise, associations, lieux de culte en particulier), ce qui peut selon le cas leur être reproché ou, au contraire, leur être accolé pour montrer leur capacité à s'intégrer dans la vie sociale et économique. 
10 Dossier Les Turcs en France : quels ancrages? I

Tableau 1 : répartition géographique des immigrés tures

et des autres étrangers en France

\begin{tabular}{|c|c|c|}
\hline RÉGION & IMMIGRÉS TURCS & AUTRES ÉTRANGERS \\
\hline Alsace & 23077 & 106370 \\
\hline Aquitaine & 3367 & 89454 \\
\hline Auvergne & 3361 & 35676 \\
\hline Basse-Normandie & 2658 & 19967 \\
\hline Bourgogne & 4416 & 50663 \\
\hline Bretagne & 4343 & 39086 \\
\hline Centre & 9209 & 83285 \\
\hline Champagne-Ardenne & 3509 & 42679 \\
\hline Corse & 9 & 19614 \\
\hline Franche-Comté & 7740 & 45087 \\
\hline Haute-Normandie & 3324 & 45814 \\
\hline Île-de-France & 48840 & 1291831 \\
\hline Languedoc-Roussillon & 3417 & 119910 \\
\hline Limousin & 2217 & 18217 \\
\hline Lorraine & 12215 & 103465 \\
\hline Midi-Pyrénées & 2259 & 97144 \\
\hline Nord-Pas-de-Calais & 2626 & 114135 \\
\hline Pays de la Loire & 3394 & 47513 \\
\hline Picardie & 4098 & 54598 \\
\hline Poitou-Charentes & 779 & 25554 \\
\hline Provence-Alpes-Côte-d'Azur & 6503 & 301197 \\
\hline Rhône-Alpes & 33629 & 330599 \\
\hline Guadeloupe & 5 & 19506 \\
\hline Martinique & 4 & 5587 \\
\hline Guyane & 1 & 27120 \\
\hline Réunion & 8 & 7147 \\
\hline $\begin{array}{l}\text { St Pierre \& Miquelon / } \\
\text { Mayotte }\end{array}$ & -- & 14 \\
\hline Total France & 185008 & 3142232 \\
\hline \multicolumn{3}{|c|}{$\begin{array}{l}\text { Source : DIYIH 2005-2006 (2007): 1.4.1.2. (p. 120), selon le ministère français de l'Intérieur. } \\
\text { Situation au } 31 \text { décembre 2006. Il s'agit des détenteurs de titres de séjour de plus de } 18 \text { ans } \\
\text { (16-18 ans travaillant et ressortissants français exclus). }\end{array}$} \\
\hline
\end{tabular}




\section{Des flux inégalement reconnus et un bilan pas toujours facile à établir}

Combien de Turcs vivent en France? La question, apparemment anodine, ne l'est pas tant que cela. Le Recensement général de la population a été abandonné au profit d'une enquête par sondage permanent dont l'inconvénient majeur est d'écraser les faibles effectifs. Or les immigrés turcs ne sont que 206000 en 1999 et 222000 en 2004-2006 ${ }^{(2)}$, représentant 6,3\% des étrangers, mais avec un taux de croissance intercensitaire de 7,8 \%. C'est l'un des rares taux croissants, avec les Africains subsahariens et les ressortissants de l'UE autres que portugais, espagnols et italiens. Pour la même période, 423271 Turcs sont comptabilisés en France par le ministère turc du Travail et de la Sécurité sociale. Ses données reposent sur les statistiques françaises (ici les titres de séjour et les recensements) et les mouvements enregistrés dans les consulats ${ }^{(3)}$. Si, logiquement, les autorités françaises appliquent sur leur territoire leurs définitions, très logiquement aussi, les autorités turques appliquent les leurs et les différences sont surtout le fait de l'appréhension de la catégorie "double nationalité".

Contrairement à ce que l'on prétend souvent, l'Alsace n'arrive qu'en troisième position, derrière l'Île-de-France et la région Rhône-Alpes. Mais, parce qu'elle est une petite région densément peuplée tout en gardant un caractère rural affirmé (dans les paysages, pas dans les statistiques), l'Alsace est sans doute la région où l'immigration turque reste la plus visible.

\section{Entrées et sorties du territoire}

8 à 10000 ressortissants turcs entrent chaque année en France à titre permanent (8 sur 10) ou temporaire (2 sur 10), indépendamment des flux touristiques. Les naissances d'enfants türkiye'li par leurs parents dépassent encore chaque année le chiffre de 7000 (7 246 en 2006) $)^{(4)}$. Ces enfants, turcs par définition si leurs parents les ont inscrits à la naissance au consulat, sont dans leur très grande majorité de nationalité française, car nés en France. La présence turque s'affirme donc chaque année, mais ce constat n'a rien de si évident. La binationalité de facto est très courante, à défaut d'être systématique, et pose souvent des problèmes cocasses aux garçons en raison du service militaire en Turquie même lorsqu'ils sont de nationalité française.

Au tableau 2 doit être accolé celui de l'immigration temporaire, car si les "bénéficiaires" ne sont pas censés rester, certains ne repartiront pas, comme dans le cas des demandeurs d'asile ayant acquis un statut de réfugié, ou celui d'étudiants qui au terme de leurs études se marient et/ou trouvent un emploi en France. 
12 Dossier Les Turcs en France : quels ancrages? I

Tableau 2 : immigration à caractère permanent en 2006. Ressortissants de pays tiers (Turquie)

\begin{tabular}{|c|c|c|c|}
\hline TYPE D'ENTRÉE & AUTRE ASIE & TURQUIE & ENSEMBLE \\
\hline Travail (salariés) & 998 & 431 & 9997 \\
\hline Travail (non-salariés) & 28 & 18 & 351 \\
\hline Refuge (statutaire) & 593 & 498 & 6800 \\
\hline Refuge (subsidiaire) & 51 & 6 & 554 \\
\hline Famille (regroupée) & 2360 & 1991 & 18140 \\
\hline Famille de Français & 4553 & 3269 & 57995 \\
\hline $\begin{array}{l}\text { Famille de réfugiés- } \\
\text { apatrides }\end{array}$ & 395 & 371 & 1534 \\
\hline $\begin{array}{l}\text { Lien } \\
\text { personnel/familial }\end{array}$ & 1509 & 1105 & 22041 \\
\hline $\begin{array}{l}\text { Autres VPF (vie privée } \\
\text { et familiale) }\end{array}$ & 505 & 306 & 6134 \\
\hline Rente & 1 & 1 & 29 \\
\hline Étrangers malades & 198 & 148 & 5854 \\
\hline Anciens combattants & 3 & 2 & 323 \\
\hline Autres & 1 & 0 & 50 \\
\hline Visiteurs (y compris actifs) & 550 & 114 & 5320 \\
\hline Total repérages & 11745 & 8260 & 135122 \\
\hline
\end{tabular}

Tableau 3 : Immigration à caractère temporaire en 2006. Ressortissants de pays tiers (Turquie)

\begin{tabular}{|lcrc|}
\hline TYPE D'ENTRÉE & AUTRE ASIE & TURQUIE & ENSEMBLE \\
\hline $\begin{array}{l}\text { APT (Autorisation } \\
\text { temporaire de travail) }\end{array}$ & 529 & 96 & $\mathbf{1 0 6 7 7}$ \\
\hline Stagiaires & 12 & 2 & 491 \\
\hline Étudiants & $\mathbf{3 1 9 4}$ & 499 & $\mathbf{4 7 2 8 6}$ \\
\hline Demandeurs d'asile & $\mathbf{2 8 3 0}$ & $\mathbf{2 5 7 0}$ & $\mathbf{2 6} 269^{*}$ \\
\hline Total repérages & 6565 & $\mathbf{3 1 6 7}$ & $\mathbf{8 4 7 2 3}$ \\
\hline $\begin{array}{l}\text { Source : Corinne Régnard, tableau A1, p. 253. } \\
\text { *: auxquels s'ajoutent } 4 \text { 479 mineurs accompagnant. }\end{array}$ & & \\
\hline
\end{tabular}


À l'inverse, les "sorties" physiques (retour volontaire ou non, "aidé" ou non, décès suivi d'un rapatriement de corps) et juridiques (acquisition de la nationalité française) sont plutôt mal appréhendées. Les naturalisations sont connues, mais les différences d'interprétation entre la France et la Turquie induisent des chiffrages contradictoires. Ainsi la France ne publie-t-elle que les retours "aidés" (50 personnes et 20 membres de famille, soient 70 individus en $2006^{(5)}$ ). La Turquie déclare 493 décès la même année, probablement rapatriés pour la grande majorité d'entre eux. Les retours volontaires non déclarés sont par définition inconnus.

\section{Les mutations d'une population immigrée en voie d'intégration}

Quarante à cinquante années après les débuts de l'immigration turque en France, il est indéniable que les conditions de vie, d'emploi, d'insertion et d'intégration ont changé. Avant même le déclenchement de la présente crise, nombre d'industries de main-d'ceuvre ont massivement licencié les ouvriers non qualifiés (automobile, textile et confection, filière bois, métallurgie et sidérurgie), poussant souvent les chômeurs à créer leur propre emploi (petite restauration, atelier de confection, bûcheronnage, bâtiment...). Il ne fau0t pourtant pas réduire la création d'entreprise à cette seule dimension : beaucoup avaient un objectif d'indépendance et certains étaient au préalable qualifiés, le salariat étant alors un passage obligé pour accumuler un capital suffisant. Les enfants ont parfois été poussés à acquérir un diplôme moyen mais adapté (droit, commerce, informatique), afin de prendre la suite (import-export, distribution, mécanique auto).

La grande enquête INSEE-INED des années quatre-vingt-dix commence à dater ; une nouvelle enquête, en cours à l'INED, sous la direction de Patrick Simon, l'un des princi-

La création d'entreprise par les "originaires de Turquie" est active, diffuse, facilement repérable sur le terrain (devantures, raisons sociales). paux protagonistes de la précédente, devrait permettre d'y voir plus clair quant à certains paramètres, tels que l'usage de la langue, le choix du conjoint, la religiosité et la pratique religieuse... On peut néanmoins se reposer sur d'assez nombreuses thèses récentes (Tietze, Amiraux, Rigoni, Autant-Dorier, Aksaz, Öztürk, Irtiş-Dabbagh, Ak, Fliche, Massicard, KaragürYalçın...), malheureusement pas toujours publiées, ainsi que sur des enquêtes importantes ${ }^{(6)}$ pour avoir une idée des évolutions en cours. 


\section{Immigration turque, travail, emploi, chômage}

Alors qu'on comptait 34756 demandeurs d'emploi turcs en 1999, ils ne sont plus que 21824 en 2006, soit des taux respectivement de 7,7 \% et 5,8 \%. On constate une baisse significative de 37,2 \% pour la période. Les prévisions du FMI et de la Banque mondiale quant à l'évolution de la crise actuelle, ou les données du nouveau Pôle emploi, incitent à la prudence. De fait, le taux moyen d'activité pour la période 2003-2005 était de 55,9\% - soit de 81,1\% pour les hommes et seulement de 27,8 \% pour les femmes - contre un taux de chômage de 29,5 \% - respectivement de $22,9 \%$ et $49,8 \%$ - et un taux d'emploi de $39,4 \%$ - respectivement de $62,5 \%$ et $13,9 \%$. Il en ressort que la population immigrée turque est potentiellement largement active parce que jeune, environ trois fois plus touchée par le chômage que les Français - les naturalisés se trouvant bien plus près des moyennes nationales - et passablement déséquilibrée par un chômage féminin dû, selon les commentateurs, aux structures familiales turques qui privilégient l'image de la femme au foyer, épouse et mère ${ }^{(7)}$.

La création d'entreprise par les "originaires de Turquie" est active, diffuse, facilement repérable sur le terrain (devantures, raisons sociales). Elle permet d'insérer de nombreux enfants de migrants (parfois au détriment de la scolarité et de la formation) et des primo-arrivants (parfois au prix des droits sociaux élémentaires). Là encore, si les statistiques existent, elles sont souvent difficiles à commenter. En raison de l'interdiction de la statistique dite "ethnique", la variable "nationalité" reste dans l'immédiat partielle et ne permet guère une réflexion sur les "originaires de...". Le Recensement général de 1999 décompte 8154 ACE (Artisans, commerçants et entrepreneurs de sociétés de plus de 10 salariés) immigrés turcs ou originaires de Turquie contre 5524 en 1990. Cela représente une croissance rapide de $47,6 \%$ pour la période intercensitaire (de $25 \%$ pour le nombre d'artisans à $109 \%$ pour le nombre de commerçants). Le dynamisme est donc grand, comparable à celui des autres pays européens, mais les entreprises turques de France restent généralement de petite taille. Les entreprises de plus de 10 salariés ne représentent en 1999 que 0,4 \% de l'ensemble de la population active turque considérée ; la majorité des indépendants est donc formée d'artisans (5\%, chiffre stable) ou de commerçants (3,8 \% contre 2,1\% en 1990). Si l'on raisonne par rapport à la "population ACE globale immigrée turque", les parts respectives de l'artisanat, du commerce et des entrepreneurs de sociétés de plus de 10 salariés sont de $60 \%, 35,5 \%$ et 4,5\% en 1999, contre $70,7 \%, 25 \%$ et $4,3 \%$ en 1990 . 


\section{Résistances à l'intégration}

"L'exception turque" mise en avant par Michelle Tribalat et son équipe ${ }^{(8)}$ ne l'est qu'au regard d'une logique française qui peut être relativisée face aux autres pratiques que connaissent les émigrés turcs. S'agit-il pour autant d'une résistance irréductible à l'intégration "à la française (alafranga) ${ }^{(9)}$ " ? Rien n'est moins sûr. Les jeunes générations, massivement de nationalité française, sont francophones bien plus que turcophones, quand bien même les enfants turcs se distinguent par une plus forte propension à user du turc dans de nombreuses circonstances, ou par une plus grande fréquentation des cours ELCO (Enseignement des langues et cultures d'origine). La création d'entreprise va dans le sens d'une meilleure insertion économique. Même si certains secteurs sont très "autonomes" dans leur recrutement, ils n'en sont pas moins intégrés dans le jeu complexe de l'économie locale et nationale. C'est sans doute plus dans les domaines religieux et culturels que les résistances peuvent apparaître : dans la mise en place d'un réseau complexe d'appartenances religieuses, dans l'importation de courants idéologiques turcs en France (ce qui n'est pas nouveau, mais la coloration islamiste de certains groupes semble progresser sans qu'il soit possible de le traduire en chiffres). La puissance du paysage audiovisuel turc est sans conteste à étudier davantage : on connaît l'offre, ses origines, ses discours, aucunement son impact réel.

\section{La défense de la turcité passe par la langue}

Datant des années cinquante, le slogan nationaliste "Vatandaşş ! Türkçe konuşş !' ("Citoyen/Compatriote ! Parle turc !") fut appliqué aux minoritaires (ici les quelques Grecs de Turquie ayant échappé à "l'échange" du traité de Lausanne en 1923) accusés d'être de faux compatriotes et de défendre les puissances étrangères en Turquie. Slogan facile mais révélateur d'une inquiétude sournoise et générale : la crainte de la dissolution dans un monde hostile aux Turcs. "Türk'ün Türk'ten başka dostu yok" ("Le Turc n'a pas d'autre ami que le Turc") proclame le quotidien Hürriyet comme devise sur sa première page, mais il n'est pas seul à le penser. La langue, paradoxalement plus que la religion, est le fondement de l'identité turque (divers travaux de Louis Bazin, François Georgeon, Altan Gökalp...) ou, pourraiton dire, des identités turques. Les nationalismes tatar, kazakh, azerbaïdjanais, ouzbek ou ouïgour, pour ne citer que ceux-là, ont dès le départ mis en exergue la défense de la langue en tant que véhicule, fondement de l'identité turque. "Dilde, 
fikirde, işte birlik" ("Unité dans la langue, la pensée, le travail"), dixit un mot d'ordre tatar lancé par le journaliste et pédagogue Ismail Gasprinski, fut repris par les nationalismes turc et tatar de la période $1890-1920^{(10)}$. Ce n'est donc ni nouveau ni propre à la Turquie. Cela s'explique très certainement par le fait que tous les peuples turcophones, après de brillantes apogées, ont connu entre le XVIII ${ }^{e}$ siècle et aujourd'hui une longue période de repli, de revers politiques et militaires, d'exodes, qui ont certes amené la naissance des États actuels, mais aussi le sentiment diffus d'être détesté, trahi, abandonné, incompris, par le monde entier ${ }^{(11)}$.

L'élément le plus évident de l'identité collective, mais aussi le plus fragile, est sans doute l'usage d'une langue propre, originale, capable de rivaliser culturellement avec les autres, surtout lorsqu'elles ont un caractère international ou impérial (anglais, français, espagnol, russe, chinois...). Le turc est passé du statut de langue impériale dominante sur un immense espace à celui de langue nationale sur un espace bien plus réduit, puis, pour ce qui nous concerne ici, à celui de langue immigrée "en ghetto ${ }^{(12)}$ ", vouée à s'étioler puis à disparaître. D'où peut-être cette résistance linguistique en émigration d'une langue qui a manqué d'être réduite à un minuscule territoire (la Turquie du traité de Sèvres, 1920). Résistance renforcée sciemment par une collusion entre l'État et une société civile pour le moins sur la défensive, avec des moyens non négligeables : ELCO (Enseignement des langues et cultures d'origine) - le cible qualifie le dispositif comme le personnel enseignant -, presse écrite, radio et télédiffusion satellitaire, sites Internet... Mais, comme le démontrent amplement les nombreux tra-

L'élément le plus évident de l'identité collective, mais aussi le plus fragile, est sans doute l'usage d'une langue propre, originale, capable de rivaliser culturellement avec les autres, surtout lorsqu'elles ont un caractère intemational ou impérial (anglais, français, espagnol, russe, chinois...). vaux de notre collègue Mehmet Ali Akınc1 (2001), la situation est tout sauf simple, et il est primordial de ne pas la simplifier jusqu'à la caricature.

Autre élément devant être pris en compte (et découlant partiellement du précédent): le multiculturalisme vécu depuis des siècles à Istanbul, dans les Balkans, au Moyen-Orient et en Anatolie. Connu sous le terme de "millet", le système de gouvernement ottoman laissait de grandes plages d'autonomie culturelle et confessionnelle aux non-musulmans, faisant de la vie en société une juxtaposition de langues, de confessions et de genres de vie. Les minorités diverses et variées vivaient en (assez) bonne intelligence, en dehors des moments de crise politique. La tolérance était réelle, mais avait des limites que chacun connaissait. Le millet, ou nation au sens ethnico-religieux, préparait sans 
doute au multiculturalisme postmoderne plus qu'à la construction de l'État-nation moderne. Il est symptomatique qu'un certain parti politique turc ait dénommé son programme "Millî Görïs"ş, titre repris par des associations turques d'Europe, ou qu'un groupe religieux mette en avant la tolérance (hoşgörü) et le dialogue interreligieux comme l'union des civilisations (medeniyetler ittifakl) face au "clash des civilisations" de Huntington. En tous cas, l'étude des conditions de la vie associative, du mariage en émigration, montre que la vision ethnico-religieuse du millet ottoman reste bien prégnante et continue à s'imposer en émigration (peut-être plus facilement que dans le pays d'origine), que la Turquie n'est pas encore un véritable État-nation moderne, unitaire et démocratique, bien que les aspirations démocratiques d'une grande partie de la population soient réelles et sérieuses.

\section{L'islam turc, toujours aussi insaisissable?}

Tous les observateurs de la vie religieuse se sont rendus compte que l'islam n'est pas monolithique et que l'islam turc en particulier échappe souvent aux connaissances acquises auprès de l'islam du Maghreb. De plus, pays officiellement laïc (principe inscrit dans la Constitution), la Turquie a instauré une sorte de clergé centralisé sous le contrôle d'une présidence des Affaires religieuses placée sous la tutelle du premier ministre. De ce fait, les ministres du culte sont fonctionnaires d'État, en émigration comme en Turquie. Échappent à cette définition les militants de groupes islamistes oppositionnels, en partie rentrés dans le rang du fait des victoires électorales de l'AKP (Parti de la justice et du développement). En émigration, lié aux services de l'ambassade ou non, l'islam turc reste à la fois discret (hors incidents spécifiques comme le port têtu du voile par des collégiennes) et incomparablement efficace. Une grande part des mouvements répertoriés en Turquie a fait souche dans une Europe parfois sanctuarisée. Les activités les plus notables, au-delà du culte, des fêtes religieuses et de la gestion des lieux (plus souvent friches industrielles rénovées que véritables mosquées), touchent le dialogue interreligieux, les femmes et l'éducation des enfants (du cours coranique à l'aide au devoir), avec un véritable investissement à long terme sur l'éducation, porté en particulier par le mouvement nurcu de Fethullah Gülen - mouvement d'une grande souplesse et adaptabilité face aux États d'immigration (des États-Unis à l'Australie, en passant par les Républiques turcophones d'Asie centrale $\left.{ }^{(13)}\right)-$, mais pas seulement. Les Süleymancı décrits par Caymaz ${ }^{(14)}$ investissent également ce champ (camps de vacances, internats, cours du soir). Loin d'être seuls, ces mouvements s'allient ou s'opposent à d'autres selon les cas, le point commun étant celui 
d'une autonomie presque totale face aux autorités françaises. Parmi les confessions et mouvements importés figurent également des groupes minoritaires comme les Assyro-Chaldéens chrétiens, bien connus dans la région parisienne, ou les alévis, plus engagés généralement dans la vie politique des pays d'immigration ${ }^{(15)}$, au moins jusqu'à ces derniers temps : les récentes élections municipales ont été largement investies par de nombreux candidats d'origine turque.

\section{Le mariage turc, dernier rempart contre l'intégration ?}

Au-delà de polémiques parfois révélatrices d'enjeux bien éloignés du sujet premier, rappelons tout d'abord que les diverses formes du mariage traditionnel turc relèvent surtout (mais pas uniquement) du mariage arrangé, forme d'alliance la plus courante dans le monde rural autour de la planète. Mariage arrangé ne signifie pas automatiquement mariage forcé, mais la limite entre ce qui relève de la tradition et ce qui renvoie à un acte délictuel ou criminel (viol en l'occurrence !) n'est pas toujours facile à tracer. Or la société turque, encore massivement rurale au plus fort de l'émigration durant la période 1960-1980, si elle est aujourd'hui majoritairement urbaine, est encore dans une période de transition. C'est ici l'individuation (au sens sociologique) et l'individualisation de la personne, fille ou femme, mais aussi garçon et homme, qui est en cause, et au-delà l'adhésion à un modèle démocratique occidental de type européen. Le constat est celui, statistique, de la permanence du mariage arrangé, parfois même plus souvent qu'en Turquie, entre cousins germains (avec deux tendances opposées) : d'une part, le mariage entre cousins issus de frères, jugé incestueux selon la logique catholique, appelé "mariage arabe", mais relevant en fait plus d'un modèle méditerranéen qu'arabe, et, d'autre part, le mariage entre cousins issus de sceurs, appelé "mariage asiatique", parce que pratiqué en Chine, en Mongolie, au Vietnam..., et relevant pour les Turcs de la tradition altaïque ccommune aux peuples turcophones et mongolophones. Le constat social est le suivant : multiplication des divorces, cas (rares, mais toujours trop nombreux) de crimes dits "d'honneur", lorsque la jeune fille ou la jeune épouse a "fauté", c'est-à-dire lorsqu'elle ne s'est pas soumise à ladite "tradition". Sujet délicat s'il en est, parce qu'il touche au plus intime et au plus sacré, dès lors que l'on mêle anthropologie et valeurs religieuses, le mariage met en jeu le système de reproduction des normes sociales, au propre comme au figuré, à travers la naissance des enfants et la défense des valeurs de la société d'origine. 
La population turque de France qui, rappelons-le une fois encore, est de plus en plus massivement de nationalité française, est-elle intégrable dans la société française ? Voilà une question qui nous renvoie à une autre question du même type : l'islam est-il soluble dans la République? Les réponses ne sont ni faciles, ni tranchées ; elles sont très proches d'une autre question tout aussi polémique, peutêtre en France plus qu'ailleurs en Europe : la Turquie a-t-elle sa place dans l'Union européenne? En réalité, ces questions apparaissent, aujourd'hui bien plus qu'autrefois, liées malgré le fait qu'elles soient pourtant, socialement, politiquement, juridiquement, dissociées. Turquie et Turcs semblent faire partie d'un inconscient collectif profond, souvent viscéral plus que logique. Ils sont aussi les objets d'enjeux électoraux et idéologiques qui les dépassent. Il serait extrêmement préjudiciable, tant pour les Français originaires de Turquie que pour l'ensemble de la population, que toutes ces questions, quelle qu'en soit l'importance réelle, restent indissociées dans l'opinion alors que de si nombreux individus et familles ont fait tant d'efforts pour s'adapter à leur nouvelle vie.

\section{Références bibliographiques}

- Samim Akgönül, Muharrem Koç, Michel Maffessoli, Stéphane de Tapia (eds), Quarante ans de présence turque en Alsace, Strasbourg, Néothèque, 2009.

- Mehmet-Ali Akınci, Développement des compétences narratives des enfants bilingues turc-français en France âgés de 5 à 10 ans, Munich, LINCOM Studies in Language Acquisition, 2001.

- Benoît Fliche, Odyssées turques. Les migrations d'un village anatolien, Paris, CNRS Éditions, 2007.

- Anne-Yvonne Guillou, Stéphane de Tapia, Martine Wadbled (eds), Migrations turques dans un monde globalisé. Le poids du local, Rennes, Presses universitaires de Rennes, 2007.

- Ahmet İçduygu, Stéphane de Tapia (eds), "Turquie 2006 : aux portes de l'Union européenne”, Revue européenne des migrations internationales, vol. 22, $\mathrm{n}^{\circ} 3,2006$.

- Verda İrtiş-Dabbagh, Les jeunes issus de l'immigration de Turquie en France, Paris, L'Harmattan, 2003.

- Gaye Petek-Şalom, Les femmes immigrées originaires de Turquie en France : nouvelles problématiques, nouveaux conflits, in Mohammed Charef (coord.), Les migrations au féminin, Agadir, Sud Contact, 2002.

- Isabelle Rigoni, Mobilisations et enjeux des migrations de Turquie en Europe de l'Ouest, Paris, L'Harmattan, 2001.

- Françoise Rollan, Benoît Sourou, Les migrants turcs de France. Entre repli et ouverture, Bordeaux, Maison des Sciences de l'homme d'Aquitaine, 2006.

- Stéphane de Tapia, Migrations et diasporas turques. Circulation migratoire et continuité territoriale, Paris-Istanbul, Maisonneuve \& Larose/IFEA, 2005.

- Emmanuel Todd, Le destin des immigrés. Assimilation et ségrégation dans les démocraties occidentales, Paris, Seuil, 1994. 


\section{Notes}

1. Débat aujourd'hui très vif, surtout depuis l'intervention du chef de l'état-major des Armées critiquant la notion de "Türkiye'li": "Türkiye'li mi, Türk halkı mı?"

2. Corinne Régnard, Immigration et présence étrangère en France en 2003, rapport annuel de la DPM, Paris, ministère de l'Emploi, de la Cohésion sociale et du Logement, Paris, DPM, 2005, p. 171.

3. DİYİH- Dıș İlişkiler ve Yurtdıșı İșçiler Hizmetleri Genel Müdürlüğü, 2005-2006 Yılı Raporu. Yurtdışındaki Vatandaşlarımıza Illişkin Gelismeler ve Sayısal Bilgiler, TC Çalışma ve Sosyal Güvenlik Bakanlığı, Ankara, 2007.

4. Ibidem, p.121.

5. Corinne Régnard, Immigration et présence étrangère en France en 2005, rapport annuel de la DPM, Paris, ministère de l'Emploi, de la Cohésion sociale et du Logement, Paris, DPM, 2007, p. 150-151.

6. Ayhan Kaya, Ferhat Kentel, Euro-Turks. A Bridge or a Breach between Turkey and the European Union. A Comparative Study of German-Turks and French-Turks, Bruxelles, Centre for European Policy Studies, 2005. En version turque : Euro-Türkler. Türkiye ile Avrupa Birligi Arasinda Köprü mü, Engel mi?, Istanbul, Istanbul Bilgi Üniversitesi, 2005.

7. Corinne Régnard, Immigration et présence étrangère en France en 2005, op.cit., p. 194-207

8. Voir Michèle Tribalat, Faire France : une enquête sur les immigrés et leurs enfants, Paris, La Découverte, 1995 ; Michèle Tribalat, Patrick Simon, De l'immigration à l'assimilation. Enquête sur les populations d'origine étrangère en France, Paris, La Découverte/INED, 1996.

9. En turc, alafranga ("à la franque"), opposé à alaturka ("à la turque"), signifie efficacité, organisation, avec une autocritique parfois féroce : tout ce qui est alaturka laisse à désirer. L'étymologie des deux termes n'étant évidemment pas turque, voilà bien un cas de représentation importée!

10. Ismail de Gaspra (d'où en russe, Gasprinski), intellectuel tatar criméen, fondateur du journal Tercüman (L'Interprète - un titre repris en Turquie), a surtout insisté sur l'enseignement et la pédagogie de la langue, en réaction à la russification dans l'empire des Romanov. Des écoles dites "nouvelles" (djadid) ont été fondées dans tous les pays de langue turque, de l'Ukraine au Turkestan et à la Sibérie, en opposition à l'enseignement traditionnel ou ancien (qadym) pratiqué dans les medersas (écoles coraniques). L'influence de cet intellectuel, somme toute de rang très modeste, a été immense dans tout l'Empire russe, mais elle a été aussi importée en Turquie ottomane par des intellectuels tatar, azerbaïdjanais et turkestanais réfugiés en Turquie. Certains d'entre eux ont même été députés de la première Assemblée nationale turque, à l'époque d'Atatürk. Défense et promotion de la langue sont l'un des fondements du nationalisme turc, comme elle l'ont été pour tous les nationalismes turciques et panturquistes.

11. Il suffit de suivre les débats passionnés et houleux qui ont suivi la tournée triomphale du président Obama en Turquie. Ce voyage, qui s'est déroulé dans d'excellentes conditions, a déclenché une polémique dans les deux jours qui ont suivi, du fait d'une petite phrase demandant l'ouverture d'un poste frontière avec l'Arménie. Second épisode le 24 avril, lorsque le président Obama, sans prononcer le mot "génocide", a reconnu celui-ci en le qualifiant, en arménien, de "grande catastrophe". Depuis, il n'y pas une soirée sans que plusieurs chaînes n'organisent en parallèle un débat sur la "réalité ou non du génocide de 1915".

12. Gaye Petek-ŞSalom, "Le turc : une langue en ghetto", in Geneviève Vermes, Vingt-cinq communautés linguistiques de la France, Paris, L'Harmattan, 1989, p. 301-316.

13. Bayram Balci, Missionnaires de l'islam en Asie centrale. Les écoles turques de Fethullah Gülen, Paris-Istanbul, Maisonneuve \& Larose/IFEA, 2003.

14. Birol Caymaz, Les mouvements islamiques turcs à Paris, Paris, L'Harmattan, 2002.

15. Elise Massicard, L'Autre Turquie. Le mouvement aléviste et ses territoires, PUF (Proche-Orient), Paris, 2005. 